\title{
The decentered magnetic dipole: a challenge for modelling
}

\author{
Ewald Gerth ${ }^{1}$ and Yurij V. Glagolevskij ${ }^{2}$ \\ ${ }^{1}$ D-14471 Potsdam, Gontardstr. 130, Germany \\ email: ewald-gerth@t-online.de \\ ${ }^{2}$ Special Astrophysical Observatory, Nizhnij Arkhyz, Karachaevo-Cherkesia, 369167 Russia. \\ email: glagol@sao.ru
}

\begin{abstract}
The global bipolar structure of the magnetic surface field is asymmetric for many stars, making its analytical description by an expansion of spherical harmonics problematic because of the coordinates. Landstreet (1970) proposed that the asymmetry could be understood assuming a decentered magnetic dipole in the stellar interior. Its surface field can be calculated for different arrangements inside and outside the star using the Magnetic Charge Distribution method. We demonstrate the effect for the two cases of the shift of the dipole along and perpendicular to the radial direction.
\end{abstract}

Keywords. Methods: analytical, stars: magnetic field

\section{Introduction}

A magnetic dipole reveals itself by the modulation of the observed integrated magnetic field strength with the rotation of the star as an oblique rotator, defining the magnetic poles as topographic features fixed to the surface of the star. Assuming a central dipole, the phase curve of the integrated magnetic field strength would be a sinusoidal one. Landstreet (1970) explained the observed deviations by the heuristic assumption of a decentered magnetic dipole, which enables one to describe most of the observed magnetic phase curves rather well. Deutsch $(1954,1955)$ was the first to represent the surface field analytically by a truncated expansion of spherical harmonics, which can be related to central magnetic dipoles, quadrupoles, etc. The description of the surface field of a decentered dipole would require such an expansion. However, since for every degree $n$ the number of parameters grows with $2 n+1$, we have for a dipole 3 parameters, for dipole+quadrupole 8 parameters, etc. Truncating the series after the octupole term yields 21 parameters. For modelling these parameters are taken as generating magnitudes. This is, indeed, a challenge for modelling!

There exist mathematical derivations for the expansion of the decentered dipole with spherical harmonics. However, the direct way using the origin of the magnetic dipole as a generating magnitude on the physical basis of potential theory and on the mathematical basis of vector algebra is recommended instead. Besides other advantages, this is the most effective way for computer calculations using the method of the Magnetic Charge Distribution (MCD) of Gerth \& Glagolewskij (2000, 2001). We demonstrate the effects of shifts of a decentered dipole along and perpendicular to the radius.

Fig. 1 demonstrates the effect of the shift of the magnetic dipole from the center of the star along the radius for $r=0,0.25,0.5,0.75,1$, and 2 directed to $\delta=45^{\circ}$ in an outward sense. Dotted lines in the magnetic Mercator maps mark regions with negative polarity. The phase curves are calculated for the inclination angles $i=45^{\circ}, 90^{\circ}$, and $135^{\circ}$, the progression of which is at maximum by phase $P=0.25$ from top to bottom. 


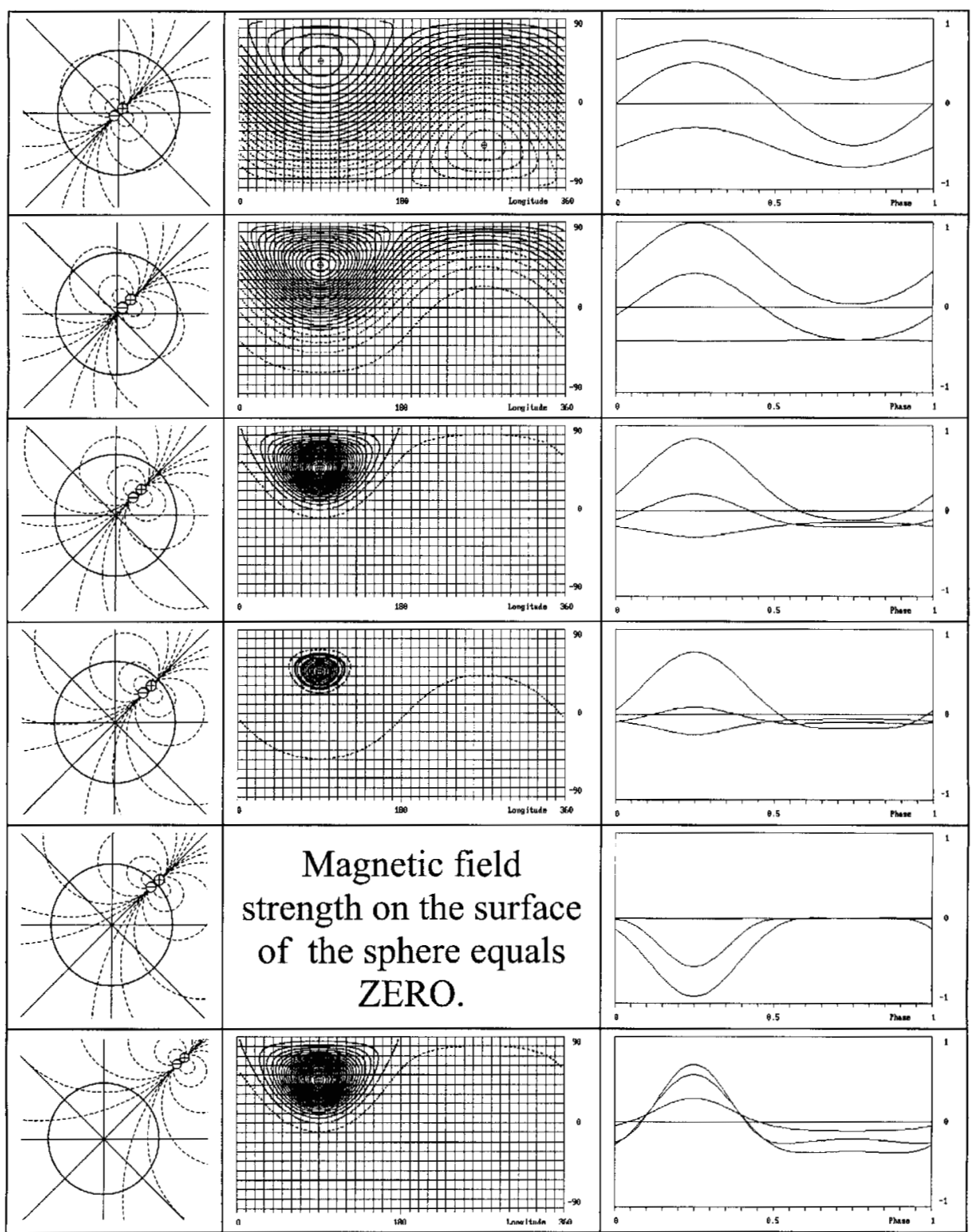

Figure 1. A series of magnetic dipoles+fields displaced along the radius by $\delta=45^{\circ}$. Left frames: Some arrangements of the dipole. Middle frames: Mercator maps of the surface field. Right frames: Phase curves of the integrated longitudinal magnetic field strength.

Comparing Fig. 2 with Fig. 1, we see that the perpendicularly arranged magnetic dipoles tend to create double poles like sunspots with increasing displacement. The field of a flatly sited dipole can be seen only from the polar side, so that the integrated field strength in the second half of the phase curve disappears. In Fig. 1 the corresponding radially directed dipole produces at the surface everywhere a zero field strength. The passage of the dipole through the surface changes the direction of the lines of force and the polarity. 


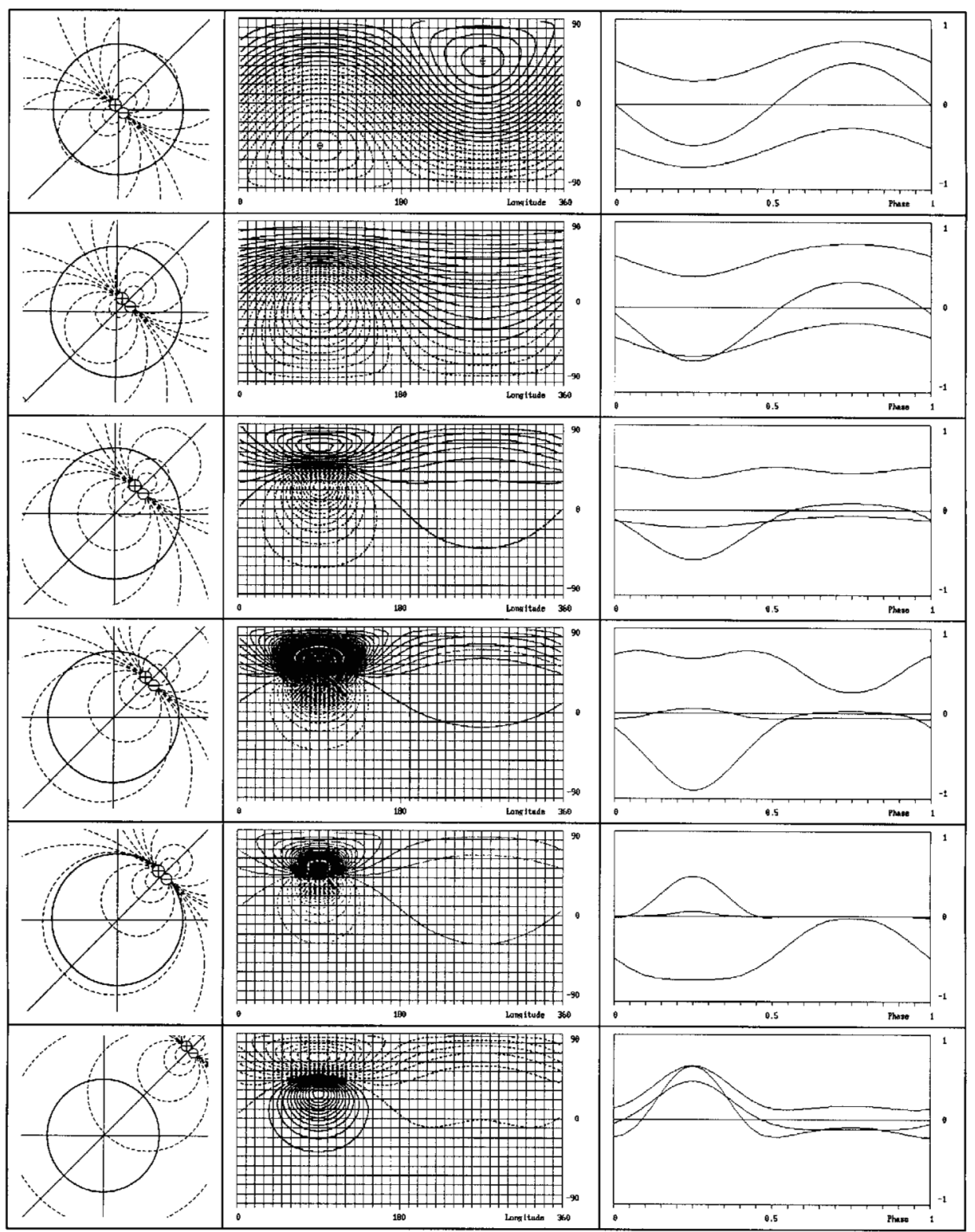

Figure 2. A series of magnetic dipoles displaced perpendicularly to those in Fig. 1.

\section{Questions and answers concerning the decentered magnetic dipole}

\section{What is a decentered magnetic dipole in a star?}

- A decentered magnetic dipole reveals itself by two asymmetric magnetic hemispheres of opposite polarity at the star's surface.

- Despite that the magnetic field is generated by an electric current circulating in the star's interior, the closed field lines penetrating the surface can be traced back approximately to point-like virtual sources bearing "magnetic charges".

- Two magnetic charges, $Q_{1}=+Q$ and $Q_{2}=-Q$, of equal magnitude but opposite 
polarity and a spatial distance $\boldsymbol{l}$ constitute the axial vector of the magnetic moment $\boldsymbol{M}$ $=Q \boldsymbol{l}$, which is the generating magnitude of the characteristic spatial dipole field.

- The magnetic dipole is defined by 7 parameters: the charge $Q$ and 3 spatial coordinates each for both sources. For $l \longrightarrow 0$ the dipole moment needs only 6 parameters.

\section{Why do asymmetric stellar magnetic surface fields occur?}

- The star has a history with disturbances (impacts, outbursts, star-spots, etc.).

- The star is affected by a cosmic neighbor in a binary system, which distorts the structure of its own magnetic field, or/and it is externally influenced by the interaction with a self-magnetic companion (e.g., white dwarf, black hole, cataclysmic system, etc.).

- The observed magnetic field strength is measured by the Zeeman-displacement of the spectral lines of the light-absorbing elements in the atmosphere, which are distributed inhomogenously over the star's surface, covering also the magnetic poles differently.

- The surroundings of the star might be partly obscured (clouds, ecliptic companion).

\section{How does one analyze the observed magnetic surface distribution?}

- The direct inversion gives an analytical representation without interpretation.

- Modelling with hypothetical parameters and fitting to the observations by a straightforward calculation on grounds of a reasonable theory is possible in any case.

- A computer program for "magnetic modelling" should have a minimum of parameters to be varied for fitting, which is guaranteed by using generating magnitudes (sources), but not for derived or ambiguous ones (e.g., field strengths at the poles).

- The parameters can be regarded as the characteristic magnitudes of a star, which describe at once the whole magnetic field and can be refined by further observation.

\section{Is the decentered dipole restricted to asymmetric stellar fields?}

- The decentered magnetic dipole is a generating magnitude itself and can be combined into a conglomerate of numerous elementary dipoles by superposing all single fields to a general one (e.g., a metallic iron magnet consisting of a vast amount of molecular magnets).

- Each elementary dipole is displaced to the zero-point of the coordinate system.

- The magnetic field of the decentered dipole(s) fills the entire space and can be calculated for any plane, whereas spherical harmonics are restricted to the sphere.

- Any magnetic field structure is represented by a sum of the fields of elementary dipoles, giving a counterpart to an expansion of spherical harmonics, but being much more general and physically founded, because it relates to the origin of the field, its sources. Thus, the modelling of the decentered magnetic dipole should not be a challenge!

\section{References}

Deutsch A.J., 1954, TIAU, Cambridge University Press, 8, 801

Deutsch, A. J., 1955, Annales d'Astrophysique 18, 1

Gerth, E., Glagolevskij, Yu.V. 2000, in Stellar Magnetic Fields (eds. Yu.V. Glagolevskij, I.I. Romanyuk), Nizhnyj Arkhyz, 151

Gerth E., Glagolevskij, Yu.V 2001, in Magnetic fields across the Hertzsprung-Russell diagram, (eds. G. Mathys, S.K. Solanki, D.T. Wickramsinghe), Santiago, Chile, 248, 333

Landstreet J.D., 1970, ApJ 159, 1001 